\title{
Morphological aspects of the pulmonary veins
}

Discipline of anatomy, Department I - preclinical disciplines, Faculty of medicine, University "Ovidius" Constanţa

\begin{abstract}
Our study was performed on 21 cases, of which 12 were plastic casts and 9 were $\mathrm{CT}$ reconstruction. We found that, most frequently ( $68.75 \%$ of the cases), the pulmonary veins were formed by two roots, one superior and one inferior. In $28.13 \%$ of cases, the pulmonary veins were formed from three roots (superior, middle and inferior) and in only one case the superior right pulmonary vein had five roots. The venous roots confluence to form the corresponding trunk was at a distance of between $0.5-2 \mathrm{~cm}$. The termination of the superior pulmonary veins was on the upper part of the anterior atrial wall, most commonly in its lateral side. The distance between the right pulmonary veins was between $0.4-3 \mathrm{~cm}$. The inferior pulmonary veins ended on the infero-lateral part of the posterior atrial wall. The distance between the left pulmonary veins was $1.2-3.4 \mathrm{~cm}$. Among the variation in number of the pulmonary veins we met: three cases with three right pulmonary veins and one case when the inferior right pulmonary vein joined terminally the inferior left pulmonary vein, forming a single venous trunk. Supplementary pulmonary veins were encountered only on the right; in one case there
\end{abstract}

\section{Dr. Iliescu D.M.}

Department of Anatomy, Faculty of medicine, University "Ovidius" Constanţa, Romania

Aleea Universitatii, Nr. 1, Campus B

Constanța, Romania

dan@anatomie.ro were two posterior right pulmonary veins (superior and inferior), in the second case were anterior right pulmonary veins (superior and inferior) and in a third case were three veins, superior, middle and inferior.

Keywords: pulmonary veins, peculiar morphology

\section{Introduction}

The pulmonary veins carry oxygenated blood to the left atrium. They are formed from three capillary networks: perialveolar (the most important, related to hematosis), peribronchial, interesting the lastbronchial ramifications (bronchopulmonary veins of Lefort) and subpleural, in relation to peripheral pulmonary lobules $[1,2,3]$. These veins, as the previous ones, end into the interlobular veins [1]. The perilobular veins join one another, forming increasingly larger trunks that finally drain into the veins at the periphery of different segments (perisegmental, subpleural and intersegmental pulmonary veins) [4]. They come together to form more voluminous vessels, so that, 
when reached the lung hilum, they form three trunks on the right lung and two trunks on the left, so one trunk for each pulmonary lobe [1]. The right middle and superior lobar veins unite, so the right lung will have two veins, superior and inferior [3,5]. Each lung veins ultimately resume to two trunks, superior and inferior, which open into the left atrium. Each pulmonary vein is composed of two roots, cranial and caudal. According to [1], the right pulmonary veins end into the left atrium close to the interatrial septum, while the left pulmonary veins end close to the lateral wall of the left atrium. According to [5], after perforating the pericardium, the pulmonary veins end in the postero-superior part of the left atrium and after [6], the right ostiums are at a distance of $25 \mathrm{~mm}$ from the left ones.

[1] founds that the two veins of a lung may join one another in a single trunk prior to their entry into the atrium (more common on the left); sometimes the three right trunks can be opened separately into the atrium, so the number of pulmonary veins increase to $3[1,5]$, to 4 or even 5 [1]; Krause [quoted by 1] presented 7 pulmonary veins for a single lung.

According to [3], there are a number of variations that particularly interest: a. the termination of the lingular vein that can be, isolated, into the superior pulmonary vein near the atrium and $b$. the coalescence, prior to their end, of the left superior and left inferior pulmonary veins, in $25 \%$ of the cases.

\section{Material and Methods}

Our study was performed on 21 cases, of which 12 were plastic casts and 9 were CT reconstruction. Plastic samples were obtained by injecting fresh lungs with Technovit 7143 , followed by $\mathrm{NaOH}$ corrosion. The reconstructions were performed in Medimar Exploration Center of the Emergency Clinical Hospital of Constanta, on a GE LightSpeed 16 Slice CT scanner. We followed: the number of roots of the pulmonary veins and their confluence into the venous trunk, their traject up to end into the left atrium, the atrial level of their ostiums and the variations in number.

\section{Results}

The number of the roots that contribute to the formation of the pulmonary veins was assessed on 32 veins, on plastic casts. We found that at the formation of the pulmonary trunk may contribute a total of 2 to 5 roots. Most commonly, in 22 cases ( $68.75 \%$ of cases), the pulmonary veins were formed by two roots, one superior and one inferior, who maintained their situation up to their confluence into the corresponding venous trunk. In 6 cases of superior pulmonary veins (18.75\% of cases), at their confluence, the two veins were placed anterior and posterior, an aspect encountered more frequently on the right, in 4 cases (12.5\% of cases). In 9 cases (28.13\% of cases), the pulmonary veins were formed from three roots (superior, middle and inferior), a variation most commonly encountered in left veins, ( 7 cases, $21.87 \%$ of cases) while for the right pulmonary veins we encountered this variant only the right inferior pulmonary vein in 2 cases $(6.25 \%$ of cases). In only one case (3.12\% of cases), the right superior pulmonary vein was formed from five roots, three roots draining the superior lobe, one the middle lobe and the fifth draining the upper part of the inferior lobe. The confluence of the roots to form the corresponding vein is made at a variable distance of the atrial wall, within the range of $0.5-2 \mathrm{~cm}$, the most common being a distance of one centimeter; the left pulmonary veins appear closer to the atrial wall. Usually, the superior root is the largest.

The traject of the pulmonary veins is specific to each vein. The right superior pulmonary vein descends, in most cases, obliquely infero-medial, sometimes approaching the vertical. Sometimes, only the superior root is vertical and joins the inferior root (horizontal or obliquely upward), forming the venous trunk which descends obliquely infero-medial. The inferior right pulmonary vein ascends obliquely 
supero-medial and, frequently, becomes horizontal near the atrium. Sometimes next to the atrium, it can describe a curve concave inferiorly, later becoming oblique infero-medial. The superior left pulmonary vein, most commonly, has a downward trajectory, oblique infero-medially and may become almost horizontal near the atrium. More rarely, it may be initially almost vertical and after it becomes oblique infero-medial. The inferior left pulmonary vein shows the most constant traject, ascending obliquely superomedial.

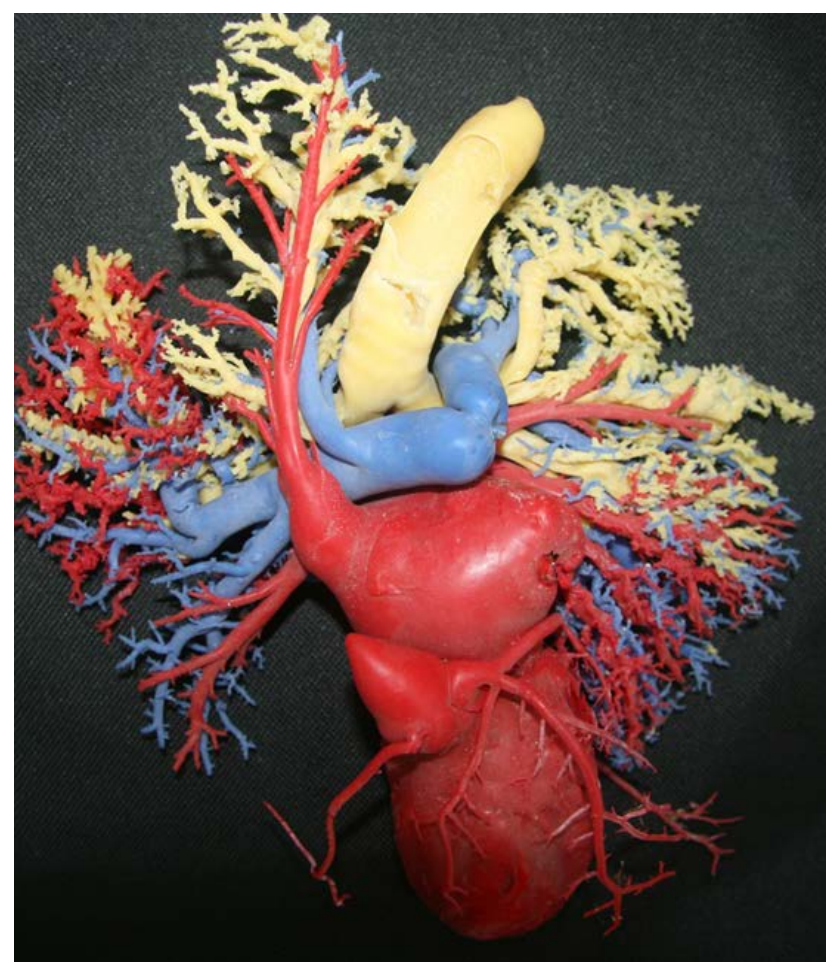

Figure 1 - Anterior view. The right superior pulmonary vein ends on the antero-supero-lateral face of the atrium. It is formed of two roots: superior and inferior. The left superior pulmonary vein is formed of two roots, at 0.5 $\mathrm{cm}$ from the atrial wall. The superior left pulmonary vein ends on the superior wall of the atrium, anterior and same level with the posterior left pulmonary vein. The distance between the right superior pulmonary vein and the left superior pulmonary vein is $2.8 \mathrm{~cm}$; the right superior pulmonary vein ends in the atrium below the left one with $1.2 \mathrm{~cm}$ and $0.8 \mathrm{~cm}$ anterior to it; red: vein; blue: artery, yellow: the trachea and bronchi.

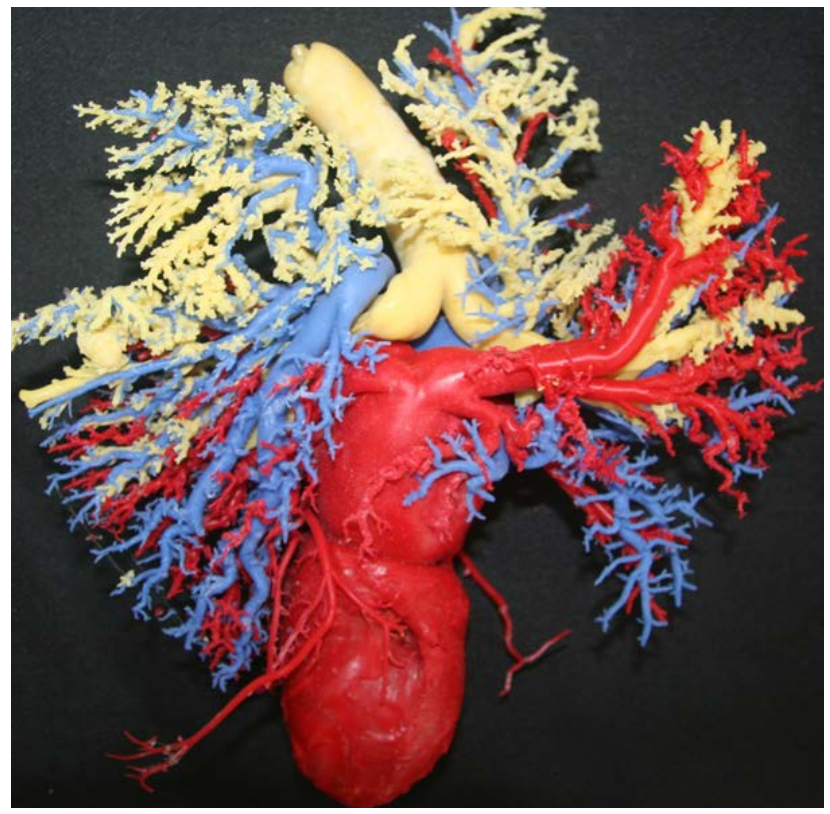

Figure 2 - Posterior view. The right inferior pulmonary vein is formed by two roots and ends into the left atrium in the middle of the posterior face, in its upper part, closer to the end of the inferior left pulmonary vein and ending higher than the superior one with $0.8 \mathrm{~cm}$.

The end of the superior pulmonary veins is on the upper part of the anterior atrial wall, most commonly in its lateral side, and sometimes same level for both superior veins, right and left, rarely closer one to another and more frequent being distanced, from 0.2 to $4.2 \mathrm{~cm}$. The distance between the right pulmonary veins, superior and inferior was 0.4 to $3 \mathrm{~cm}$. The inferior pulmonary veins showed frequent variations, mostly upon their termination, in relation to the superior ones. Typically, they end on the infero-lateral face of the posterior atrial wall, located at a level below the termination of the superior veins. We encountered 8 cases (25\% of cases) in which the inferior pulmonary vein ended above the superior pulmonary vein termination, most frequent on the left, in 6 cases (18.75\% of cases). Only on the left, in two cases (6.25\% of cases) the two pulmonary veins ended at the same level, one anterior and the other posterior and also in two cases the left and right inferior veins ended same level. The distance between the left pulmonary veins, superior and inferior, was 1.2 to $3.4 \mathrm{~cm}$. 


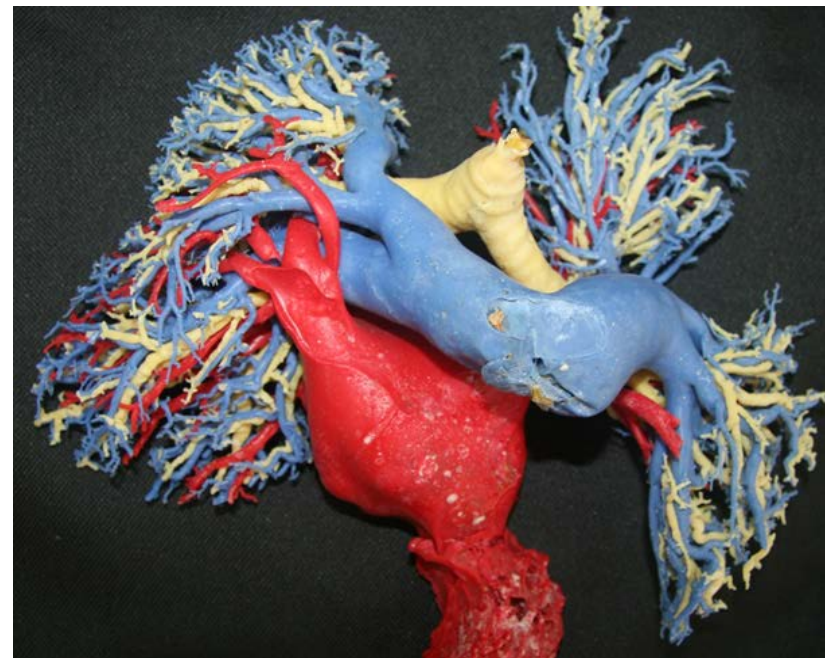

Figure 3 - Anterior view. A superior right pulmonary vein formed by 5 roots that confluence at $1.8 \mathrm{~cm}$ above the left atrium, in which ends on the right supero-lateral portion of the anterior wall. Four branches are disposed anteriorly and one is posterior to the confluence of the anterior ones. The left superior pulmonary vein ends on the supero-lateral portion of the anterior wall of the left atrium, slightly below the end of the right superior pulmonary vein. It is composed of three branches (one anterior, most voluminous and two posterior), which confluence at approximately $2 \mathrm{~cm}$ from its completion into the atrium.

Regarding the variation in number of the pulmonary veins, we encountered: three cases with three right pulmonary veins and one case when the inferior right pulmonary vein joined terminally with the inferior left pulmonary vein, forming a single trunk that ended in the left atrium.

Among the three cases with additional veins, two cases were discovered on casts of plastic injection and one case with $\mathrm{CT}$ reconstruction. In one case there were two posterior right pulmonary veins that ended on the superior part of the posterior atrial wall, one being located anterior-superior to the other. Both were located on a higher plane to the left superior pulmonary vein. First right posterior pulmonary vein (superior) drain the blood from the back of the middle lobe and the postero-superior part of the inferior lobe. The second right posterior pulmonary vein (inferior) drained blood from the posterior face of the inferior lobe, in its upper part and both sides of the inferior lobe, on its lower part. The distance between the right posterior pulmonary veins and the left superior pulmonary vein was $4.5 \mathrm{~cm}$ and the distance between the right posterior pulmonary veins and the left inferior pulmonary vein was $5.2 \mathrm{~cm}$.

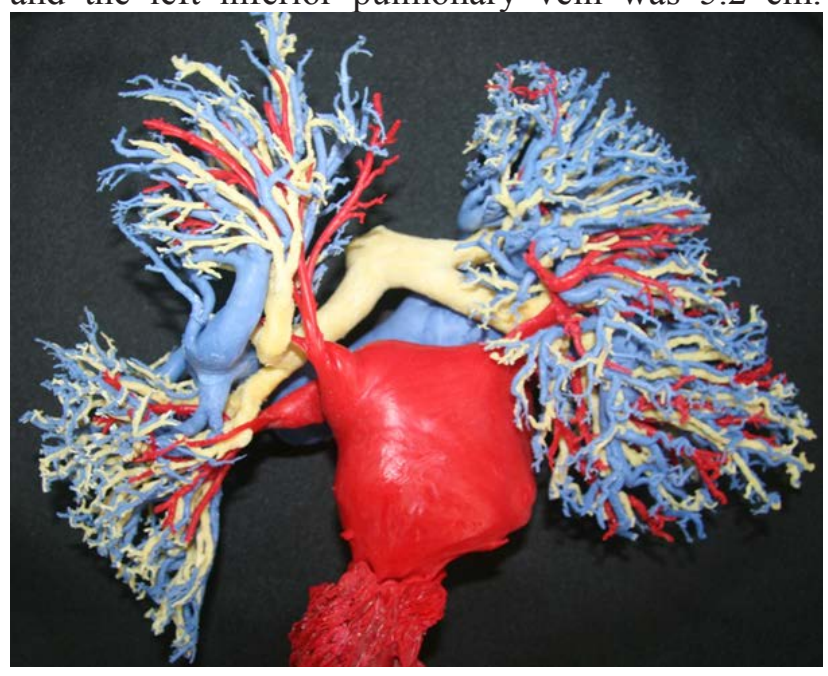

Figure 4-Posterior view. Two posterior right pulmonary veins, the superior and inferior that end of the superior portion of the posterior atrial wall, one disposed anterior and superior to the other. The distance to the superior left pulmonary vein is $4.5 \mathrm{~cm}$ and the distance up to the inferior right pulmonary vein $5.2 \mathrm{~cm}$. The inferior left pulmonary vein is placed posterior to the anterior one with $0.6 \mathrm{~cm}$ and laterally with $0.4 \mathrm{~cm}$, both ending at the same superior level. The distance between the superior right pulmonary vein and the anterior left pulmonary vein is $4.2 \mathrm{~cm}$. The inferior left pulmonary vein is formed by 2 branches at $12 \mathrm{~mm}$ before its end on the posterolateral face of the posterior atrial wall. The inferior left pulmonary vein ends into the left atrium at a higher level than the superior one.

In the second case discovered by plastic injection were two anterior right pulmonary veins, one superior and one inferior. The inferior one was formed by two roots: the medial (larger) and lateral, joined at about $3 \mathrm{~cm}$ above its termination on the infero-lateral side of the anterior atrial wall, at a distance of $3 \mathrm{~cm}$ inferior to the end of the right superior pulmonary vein. It drained the anterior part of the upper and middle lobes, and, near its end, receives a small venous trunk that drained the inferior lobe. The antero-superior right pulmonary vein, located posterior to the previous one, drained the 
right superior lobe and the anterior part of the middle lobe and ended on the supero-lateral part of the atrial wall, at $2.4 \mathrm{~cm}$ above the inferior one. In the third case, the three veins were disposed superior, middle and inferior, the superior vein (the most voluminous) draining the upper lobe, the middle vein (the thinnest) drained the middle lobe and the inferior one drained the lower lobe.

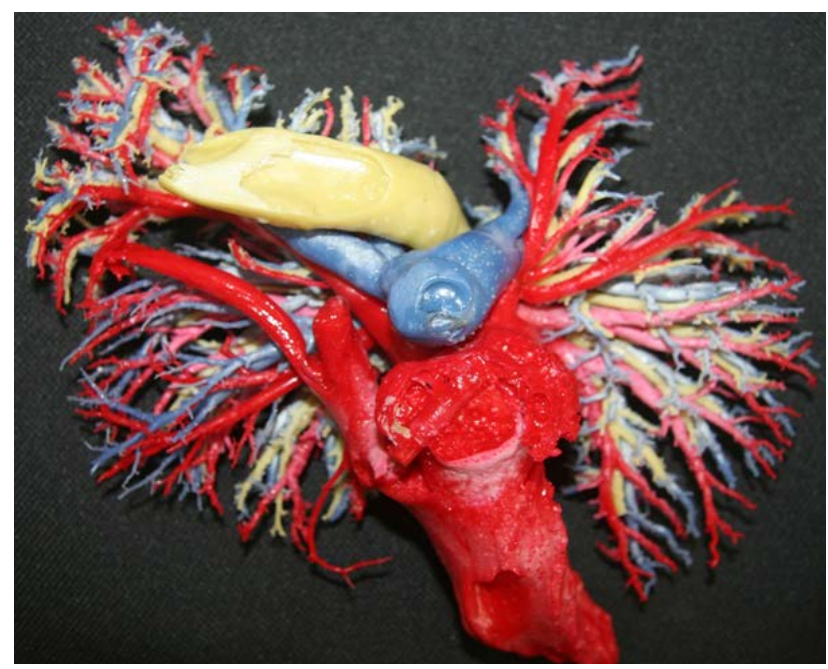

Figure 5 - Anterior view. Two anterior right pulmonary veins, one superior and another inferior. The superior left pulmonary vein is formed by 3 roots: superior, middle (the thinnest, drains blood from the lingula) and inferior; all three confluence at $8 \mathrm{~mm}$ from the atrial wall, ending on the supero-lateral part of the anterior atrial wall, prior to the common trunk of the inferior right and left pulmonary veins.

The confluence of the inferior right pulmonary vein with the inferior left pulmonary vein is made at $0.5 \mathrm{~cm}$ from the atrial wall, ending on the left superolateral part of the posterior atrial wall, slightly above the termination of the right antero-superior pulmonary vein; this case also presented two anterior right veins.

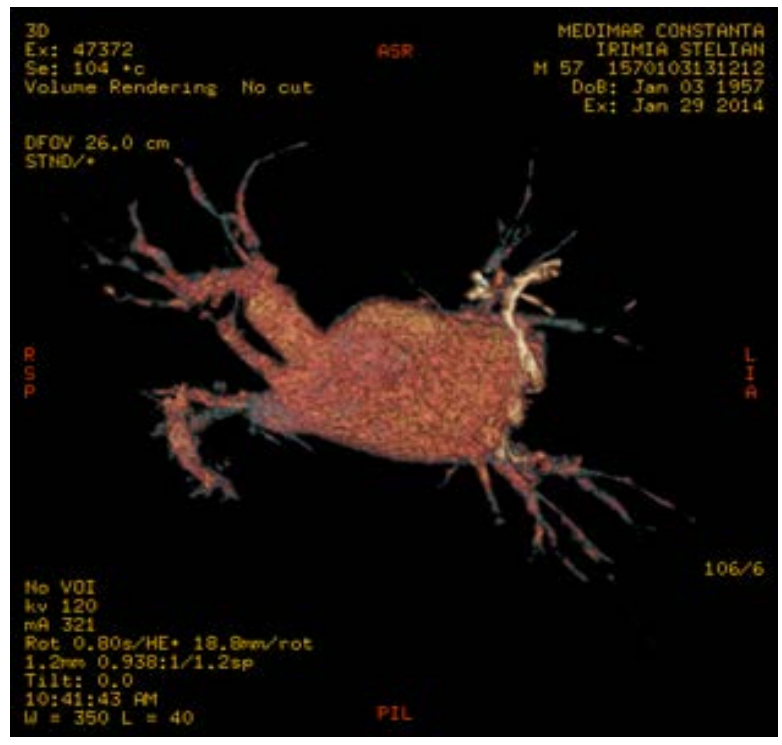

Figure 6-Three right pulmonary veins; the vein of the middle lobe ends into the left atrium as middle right pulmonary vein, equally between the superior and the inferior right pulmonary veins.

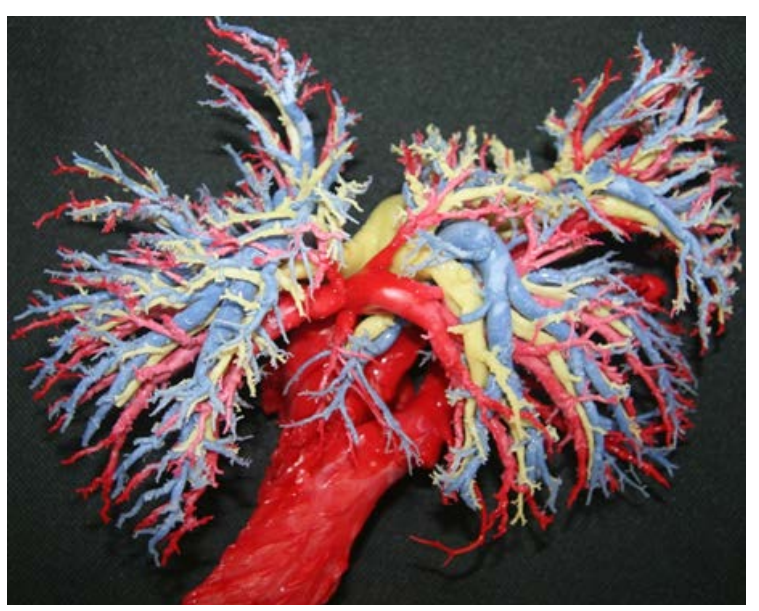

Figure 7 - Posterior view. The inferior right pulmonary vein is formed of three branches: superior, middle (most voluminous) and inferior. Terminally, confluences with the inferior left pulmonary vein, forming a single venous trunk at $0.5 \mathrm{~cm}$ from the atrial wall, ending in the left atrium slightly above the termination of the right antero - superior pulmonary vein. The distance between the superior right pulmonary vein and the superior left pulmonary vein is $2.5 \mathrm{~cm}$. The distance between the right pulmonary veins, superior and inferior, is $2.2 \mathrm{~cm}$. The distance between the superior right pulmonary vein and the inferior right pulmonary vein is $1.6 \mathrm{~cm}$. 


\section{Discussion}

Some of our results are confirming the literature, but also some data that do not correspond to the classics statistics. Regarding the number of venous roots that forms the pulmonary vein, most of the authors state that they are formed of two roots, the superior and the inferior $[1,2,3,4,5]$; according to [6] the superior right pulmonary vein is formed by four roots and the rest of the three other veins are formed by two veins. We have not found the formation of the pulmonary vein from four roots, but we found three roots for the superior pulmonary veins in a proportion of $18.75 \%$ of the cases and a single case of right superior pulmonary vein from five roots. These aspects (three or five roots for a pulmonary vein) we have not met in the literature and also the variation with both roots placed anterior and posterior roots at their confluence. [3] shows that the two roots of the pulmonary veins confluence most commonly in the anterior portion of the lung pedicle, while we found that the roots confluence near the atrial wall, especially for the left pulmonary veins. Also [3] gives the possibility that a root of the pulmonary vein drains two adjacent lobes, something that we also met, but only in cases where lung fissure was incomplete .

The pulmonary veins get their name by the lung territory they drain. In relation to their atrial termination, we found that in $25 \%$ of cases the inferior pulmonary vein ends in the atrium above the termination of the superior pulmonary vein; for the left pulmonary veins only, they end in the atrium at the same level, one superior and the other inferior, in $6.25 \%$ of cases. According to [6], the distance between the right and left pulmonary ostiums is 2.5 $\mathrm{cm}$; we found a distance of 0.1 to $4.2 \mathrm{~cm}$ for the superior veins and 0.4 to $5.2 \mathrm{~cm}$ for inferior veins, closer one another when the two veins end closer to the supero-medial portion of the posterior atrial face.

In the literature are reported multiple anatomic variations of the pulmonary veins, such as: the union of two veins into a single one [1,5], most commonly on the left; in most of those cases they were additional pulmonary veins, four or even five veins for a lung
$[1,3,5]$. Krause [quoted by 1$]$ cited seven veins. In our study, the number of supplementary pulmonary veins has been in a total of five, all on the right.

When the right and the left inferior pulmonary veins confluence, into the left atrium ended four pulmonary veins due to the fact that, in this case, there were three right pulmonary veins.

\section{Conclusions}

The pulmonary veins are less described in the literature, compared with much higher frequency of the description of the pulmonary arteries and incomparably less frequently as compared with the description of the other vessels of the body. [1] notice that the lung is the only organ that has two veins for a single artery. The knowledge of the pulmonary vein morphology and especially the existence of supernumerary vein or pulmonary vein confluence in a single trunk may be useful to avoid accidental injury within the lung surgery, to facilitate the ablation in cases of arrhythmia, thus resulting the need for compulsory evaluation of the morphology of the pulmonary veins by CT scan or MRI [7]. Various studies have demonstrated the accuracy of this integrated imaging process, in order to guide the catheter for the ablation of atrial fibrillation. The knowledge of the number and location of the pulmonary veins (PVs) is important to improve the efficacy and safety of the procedure [7].

\section{References}

1. Testut L. (1921). Traité d'anatomie humaine. Angeiologie. (pp. 265-266). Paris: Ed. Gastoin Doin

2. Bourgeon A. \& Chevrel J.P. (1994). Anatomie 
clinique. Le Tronc-Les poumons. (pp. 219-240). Paris: Ed. Springer-Verlag

3. Bouchet A. \& Cuilleret J. (1991). Anatomie topographique, descriptive et fonctionnelle. Le cou. Le torax. (pp. 1067-1076, 1117-1122, 11361140). Paris: Ed. Simep

4. Rouvière H. \& Delmas A. (1997). Anatomie Humaine. Descriptive, topographique et fonctionelle, Tome 2. (pp. 305-306). Paris: Ed. Masson

5. Standring S. (2005) Gray's Anatomy. The Anatomical Basis of Clinical Practice. (pp. 1027). Ed. Elsevier-Churchill Livingstone
6. Kamina P. (1997). Anatomie. Vol. 11. Dos et Thorax. (pp. 175-178). Paris: Ed. Maloine

7. Díaz-Caraballo E., Merino J.L. \& Guzmán G. (2010). Posterior Accessory Pulmonary Veins and Atrial Fibrillation. Rev Esp Cardiol. 63(2), 224

8. ***** Federative Committee on Anatomical Terminology. (1988). Terminologia Anatomica. International Anatomical Terminology. (pp. 9192). Stuttgart: Thieme Verlag 